\title{
The Role of Oxytocin, Vasopressin, and D-Cycloserine in Remediating Social Behavior in Rats with Amygdala Lesions
}

\author{
Kristina L. Scanlan, ${ }^{\mathrm{a}}$ Dr. Alan Gittis, ${ }^{\mathrm{b}}$ Dr. Robin McGovern ${ }^{\mathrm{a}}$
}

\begin{abstract}
Autism is a common neurodevelopmental disorder that affects communication and social behavior. To reduce the social deficits characteristic of autism, the compounds oxytocin, arginine vasopressin, D-cycloserine, and D-cycloserine + oxytocin were explored as therapeutic agents. Twenty-one Long Evans Hooded rats underwent a bilateral amygdala lesion, which reduced the time of social interactions between the pairs of animals. Upon administration of D-cycloserine, the social deficits induced by the lesions were significantly reversed in both sexes. In addition, it was observed that the efficacy of the treatments was affected by the sex of the subjects. Male rats had the largest increase in social behavior when given D-cycloserine. However, female rats experienced the largest reduction in social impairment when administered oxytocin. Thus, sexually dimorphic treatments should be further investigated for individuals with autism spectrum disorders.
\end{abstract}

Keywords: oxytocin, vasopressin, D-cycloserine, amygdala, sexual dimorphism

Autism spectrum disorders (ASDs) refer to several similar developmental conditions that include childhood disintegrative disorder, Asperger's disorder, pervasive developmental disorder not otherwise specified, and autism (4th ed., text rev.; DSM-IV-TR, American Psychiatric Association, 2001). The DSM-IV-TR (2001) lists the symptoms of these disorders as having repetitive behaviors and limited interests, rigidity towards following routines, perceiving either extreme sensitivity or a sensory deficiency towards different environmental stimuli, a lack of social and emotional reciprocity, hardships in forming and preserving relationships, and difficulty in using and understanding nonverbal behaviors. These symptoms appear in early childhood and can be so severe as to impair and restrict day-to-day functioning (4th ed., text rev.; DSM-IV-TR, American Psychiatric Association, 2001). In fact, the social behavior deficits can be the most debilitating aspect of ASDs. Unfortunately, there are limited pharmaceutical treatments available for this disorder, but new treatments for improving social behavior are currently being investigated. The neuropeptides oxytocin (OT) and arginine vasopressin (AVP) and the antibiotic D-cycloserine (DCS) are especially promising for alleviating the social deficits found in disorders like autism. This study investigated the application of using these chemical compounds as therapeutic agents for increasing social behavior.

Today, ASDs are prevalent worldwide and affect every 1 in 200 individuals (Baron-Cohen, 2002). Additionally, ASDs exhibit a gender bias in which men are four times more likely to be affected than women (Bill \& Geschwind, 2009). Although the causes of ASDs and specifically autism are not fully understood, several theories ranging from genetic factors to a dysfunction of the neuroendocrine system have been proposed. The domain of the chromosome 15q11-13 has been implicated in autism since it has one of the most common mutations present in autistic individuals (Schanen, 2006). A duplication mutation of this area affects GABA receptors
(Schanen, 2006). Schanen (2006) found that a dysregulation of GABA receptors could cause autistic-like symptoms including anxiety and social behavior deficits. However, the mutation of the chromosome domain 15q1113 only accounts for five percent of individuals with autism (Schanen, 2006). Additionally, the DLX5 gene on chromosome $7 \mathrm{q}$ may also play a role in autism as well. Since DLX5 is one of the genes responsible for regulating the expression of the enzyme glutamic acid decarboxylase, which is responsible for producing GABA, a mutation of this gene will produce an abnormal amount of the neurotransmitter (Schanen, 2006). Abnormal amounts of GABA can also result in reduced social behavior (Schanen, 2006).

A neuroendocrine system dysfunction theory for autism has also been proposed. This theory has resulted in close examination of the two related neuropeptides OT and AVP, which are both involved in social behavior and recognition (Bielsky \& Young, 2004). In Green, Fein, Modhal, Feinstein, Waterhouse, and Morris's study (2001), it was found that OT was positively correlated with the amount and quality of social relationships and everyday life skills. The study also found that in individuals with autism, OT has been found in lower than normal levels in the blood, which could account for lower than normal levels of socialization (Green et al., 2001). Furthermore, even though the autistic individuals studied had lower than normal amount of OT in their blood, there was double the concentration of the precursor form of OT in the blood as well (Green et al., 2001). Carter (2007) theorizes that the precursor of OT has a diminished function as compared to the fully functional form of OT, which could lead to less modulation of social behavior. However, a dysfunction in the machinery that converts the precursor to OT could also potentially cause lower than normal levels of blood plasma OT as well.

An AVP dysregulation has also been found to create social deficits similar to those of OT dysregulation as well. In 2005, Al-Ayadhi determined that there was a

b. Westminster College, PA 
deficiency in the blood plasma concentration of AVP in autistics when compared to normal controls. Additionally, using 'knockout mice' that lack the genes for the OT receptor and the AVP V1a receptor, have shown severe social deficits ( Bartz \& Hollander, 2008; Bielsky, Hu, Szegda, Westphal, \& Young, 2004; Bielsky \& Young, 2004; Bielsky, Hu, \& Young, 2005). Other models have been used as well to mimic the social deficits found in ASDs.

The basolateral amygdala is an associative area within the limbic system in the midbrain that is responsible for emotion, modulating social behavior in the correct social context, emotional, motor, and spatial learning, avoidance behavior, anxiety, allows for Pavlonian conditioning, and is needed for accurately identifying facial expressions ( Bachevalier \& Malkova, 2006; Cardinal, Parkinson, Hall, \& Everitt, 2002; Daenen, Wolterink, Gerrits, \& Van Ree, 2002; Davis \& Whalen, 2001). Additionally, the basolateral amygdala is an integration center for sensory information that is sent from the cortex, thalamus, and hippocampus (Davis \& Whalen, 2001). The pathway then relays information to either the orbital frontal cortex, the dorsal and ventral striatum, the lateral bed nucleus of the stria terminalis of the amygdala, the central nucleus of the amygdala, and can also relay information back to the hippocampus (Davis \& Whalen, 2001). Due to the multiple associations of the basolateral amygdala, a lesion in either one or both of the amygdalae would be detrimental other structures' functions connected to its pathway as well.

Several studies have implicated amygdala pathology in autism. In 1993, Kemper and Bauman conducted a post-mortem autism study, which examined the brains of both individuals with autism and age-matched controls (Amaral, Bauman, \& Schumann, 2003). Among the six brains of individuals diagnosed with autism, neuronal differences in the amygdala were identified, which were not present in the brains of the control (Kemper \& Bauman, 1992; Amaral, Bauman, \& Schumann, 2003). Acquired amygdala damage can also produce symptoms similar to that of ASDs. Hamann and Adolphs (1999) followed two patients that both had bilateral amygdala lesions from a herpes simplex virus. It was found that these patients not only lacked the ability to identify facial expressions, but they were unable to recognize emotions in general (Hamann \& Adolphs, 1999). The patients in Hamann and Adolphs' (1999) study showed symptoms remarkably analogous to the inability to accurately perceive emotions and facial expressions as found in autism.

In order to better understand the disorder and to find better treatment options for individuals with autism, researchers have been able to design an accurate autism model involving bilateral amygdala ibotenic acid lesions in rats to induce autistic-like deficits in social behavior (Bachevalier \& Loveland, 2006; Daenen, Wolterink, Gerrits, \& Van Ree, 2002, Gittis \& Alfieri, 2009). Social behavior in rats is defined as body contact, jumping over or running under one another, anogenital sniffing, social sniffing, cuddling, and social grooming (Daenen, Wolterink, Gerrits, \& Van Ree, 2002). When bilateral amygdala ibotenic acid lesions are present, social behavior in rats is significantly impaired, thus making this type of lesion ideal for an autism model (Gittis \& Alfieri, 2009). However, Gittis \& Alfieri (2009) was able to reverse the induced social impairment by administering $.6 \mathrm{ng} / \mathrm{kg}$ of OT to the lesioned animals. Furthermore, AVP has a similar role in reversing social impairment (Dantzer, Bluthe, Koob, \& Le Moal, 1987).

OT is a sexually dimorphic neuropeptide that is nine amino acids long and is produced in the paraventricular nucleus of the magnocellular cells of the hypothalamus (Bartz \& Hollander, 2008; Carter, 2007; Green et al., 2001; Hammock \& Young, 2006; Lim, Bielsky, \& Young, 2005). This nonapeptide is released into the circulatory system from the neurohypophysis (Bielsky \& Young, 2004). OT has various functions within the body including aiding in parturition, lactation, sexual behavior, maternal behavior, social behavior, and bonding (Bartz \& Hollander, 2008; Bielsky \& Young, 2004; Carter, 2007; Green et al., 2001). Estrogen is an essential component of OT and since this neuropeptide is sexually dimorphic, its concentration and receptor sites in the body are found in larger amounts in females than in males (Carter, 2007). Moreover, OT has only one type of receptor and is most abundant in the limbic system (Bartz $\&$ Hollander, 2008; Carter, 2007). Since females are four times less likely to develop autism than males, Carter (2007) has theorized that OT may actually help prevent females from developing autism. Additionally, Gittis \& Alfieri (2009) and Bielsky \& Young (2004) found that OT can actually promote social behavior in animal models of autism. Thus, OT may be a beneficial therapuetic agent for alieviating a social deficit.

Another promising neuropeptide for treating autism is AVP. AVP is also a sexually dimorphic nonapeptide that is from the same lineage of genes as OT (Bielsky, Hu, \& Young, 2005; Bielsky \& Young, 2004; Lim, Bielsky, \& Young, 2005). This neuropeptide is formed in the supraoptic nucleus of the hypothalamus and is released from the neurohypophysis like OT (Bielsky, Hu, Szegda, Westphal, \& Young, 2004; Bielsky \& Young, 2004). AVP is dependent on androgens like testosterone as a precursor and has a variety of responses within the body (Bielsky, Hu, \& Young, 2005). Some of AVP's functions include flank marking, affiliation, paternal behavior, aggression, maintaining blood pressure and a homeostatic fliud balance, the stress response, pair bonding, social recognition, and social behavior (Carter, 2007; Lim, Bielsky, \& Young, 2005). AVP has three G-protein receptors: the $\mathrm{V} 1 \mathrm{a}$ receptor $(\mathrm{V} 1 \mathrm{aR})$, the $\mathrm{V} 1 \mathrm{~b}$ receptor (V1bR), and the V2 receptor (Frank \& Landgraf, 2008). However, only the V1aR and V1bR have demonstrated involvement in social behavior, whereas the V2 receptor regulates fluid balance in the kidneys (Bielsky, $\mathrm{Hu}, \&$ Young, 2005; Bielsky \& Young, 2004; Frank \& Landgraf, 2008; Hammock \& Young, 2006; Lim, Bielsky, \& Young, 2005). High densities of the V1aR have been located in the medial amygdala, lateral septum, medial preoptic stria terminalis, and the bed nucleus of the stria terminalis (Bielsky \& Young, 2004; Hammock \& Young, 2006). The $\mathrm{V} 1 \mathrm{aR}$ is the most prominent AVP receptor and along with the $\mathrm{V} 1 \mathrm{~b}$, has a higher density of receptors and a larger concentration of the chemical itself in males than females (Carter, 2007; Hammock \& Young, 2006). In V1aR 
knockout mice studies (V1aRKO), severe deficits in social behavior and social recognition have been found in animals that lack the V1aR gene, further implicating it in its role in autism (Bielsky, Hu, Szegda, Westphal, \& Young, 2004; Bielsky, Hu, \& Young, 2005). The V1bR is found in the highest densitiy in the pituitary gland and is known to be involved in regulationg the stress response (Bielsky, $\mathrm{Hu}$, Szegda, Westphal, \& Young, 2004; Carter, 2007). In 2004, Bielsky, Hu, Szegda, Westphal, and Young conducted a knockout study deleting the V1bR gene (V1bRKO) in a strain of mice. The reserachers found that knocking out the V1bR gene creates only a slight social deficit, but nowhere near as dramatic as the social impairment elicited by the V1aRKO (Bielsky, Hu, Szegda, Westphal, \& Young, 2004). This research suggests that a dysfunction in the V1aR or a dysregulation of AVP circulating throughout the body could produce a social deficit similar to the symptoms of autism. This deficit has been reverersed in the V1aRKO by administering AVP to the subject, demonstrating a therapeutic benefit that may allieviate social behavior impairments (Bielsky, Hu, Szegda, Westphal, \& Young, 2004).

Originally used as a medication to treat pulmonary tuberculosis, DCS has proven to be useful in treating the symptoms found in disorders like social anxiety, schizophrenia, post-traumatic stress disorder, obsessive-compulsive disorder, drug addiction, and phobias (McDougle, Stigler, Erickson, \& Posey, 2006; Modi \& Young, 2011). Recently however, several studies have found DCS beneficial in reversing the social deficits of autism in both animal models and human subjects (Jacome, Burket, Herndon, \& Deutsch, 2011; McDougle, Stigler, Erickson, \& Posey, 2006; Modi \& Young, 2011; Otto, 2011; Posey et al., 2004). In Jacome, Burket, Herndon, \& Deutsch's study (2011), the researchers found that when DCS was administered to a socially deficient Balb/c mice before social testing, the subjects interacted with a novel mouse for a longer duration of time than when given saline. Furthermore, the Balb/c mice that were given DCS were compared to their socially normal controls, the Swiss Webster mice, it was found that the Balb/c mice interacted more with the novel mouse than the Swiss Webster control subjects. Additionally, a pilot study conducted by Posey et al. in 2004 explored the pharmaceutical benefits of DCS and had individuals with autism take $25 \mathrm{mg}$ of the drug daily. Posey et al. (2004) reported that after eight weeks of treatment, the partcipants severity of symptoms encompassing social deficits decreased by 60 percent, and 40 percent of the participants' physicians rated their patients as being 'much improved.'

The possible explanations for DCS's effect is that it has been found to interact with the N-methyl-D-asparate (NMDA) receptors in the brain by having an agonistic action and facilitates with the binding of ligands (Jacome, Burket, Herndon, \& Deutsch, 2011; McDougle, Stigler, Erickson, \& Posey, 2006; Modi \& Young, 2011). Modi \& Young (2011) and Otto (2011) proposed that DCS's ability to increase socialibility is due to DCS interacting with the areas of the brain with high densities of OT receptors such as the amygdala to facilitate OT's role in inducing social behavior. Hence, the promising results from these recent studies prompt further investigation of DCS as a treatment for the social impairments found in ASDs, especially when observing the effects of DCS + OT.

Since OT, AVP, and DCS have been shown to reduce social deficits, one would expect to see that administration of these chemical agents could be used to remediate social disorders like autism. Using a bilateral basolateral amygdala ibotenic acid lesion autism rat model, its expected that administration of OT, AVP, and DCS will increase overall social behavior after the social deficit has been induced. However, since both the OT and the AVP system are sexually dimorphic, it would be expected that in female subjects with amygdalar lesions, OT would produce a larger increase of social behavior than in male rats. Additionally, it could also be proposed that male rats with amygdalar lesions would exhibit a larger increase in social behavior when given AVP.

Method

Subjects

Twenty-one Long Evans Hooded male and female rats were used. On post-natal day (PND) 7, all subjects underwent a bilateral basolateral amygdala ibotenic acid lesion. Promptly after the lesioning surgery, the animals were placed back in their respective containers with their mother. The rats were removed from their mothers and subsequently weaned on PND 25. During the weaning phase, the subjects were housed together in same sex groups of three animals from the same litter. Food and water was available for 24 hours a day ad libitum.

\section{Materials and Methods}

\section{Surgical Tools}

Several surgical tools were used for the procedure. A stereotaxic apparatus was used for the bilateral amygdala lesion surgery. A clay holder was also used as well to facilitate keeping the subjects' heads in alignment for the stereotaxic surgery since the rats are too small on PND 7 for the ear-bars. An $1 \mathrm{~mm}$ drill bit was used in order to access the amygdalae in order to form holes in the skull. The lesions were produced by using a 10 $\mu \mathrm{L}$ syringe to deliver ibotenic acid to the amygdalae.

Drugs

Cyclo(Tyr-lle-Gln-Asn-Asu)-Pro-Leu-Gly-NH oxytocin, Cys-Tyr-Phe-Gln-Asn-Cys-Pro-Arg-Gly- $\mathrm{NH}_{2}$ [Disulfide Bridge: 1-6] arginine vasopressin, and Dcycloserine were obtained from Sigma-Aldrich. The OT was administered subcutaneously at a $0.6 \mathrm{ng} / \mathrm{kg}$ dose delivered at $0.01 \mathrm{ml} / \mathrm{g}$ body weight, the AVP was subcutaneously administered at a 50 percent solution delivered at $0.01 \mathrm{ml} / \mathrm{g}$ body weight, and DCS subcutaneously administered at $320 \mathrm{mg} / \mathrm{kg}$ dose, which was delivered at $0.01 \mathrm{ml} / \mathrm{g}$ body weight. Prior to the study, the OT, AVP, and DCS were diluted to $1 \mathrm{~mL} / \mathrm{kg}$ with phosphate-buffered saline.

\section{Procedure}

Surgery

All procedures used in this study were in line with the APA guidelines for the care and the use of the lab 
animals. Protocol for surgery was taken from Gittis \& Alfieri (2009). At PND 7, subjects were anesthetized in an ether chamber prior to bilateral amygdala ibotenic acid lesion surgery. After the rats were anesthetized, they were transferred to the stereotaxic apparatus. Once the anesthetized animals were secured in the apparatus, bilateral holes were formed in the skull using a $1 \mathrm{~mm}$ drill bit. A $10 \mu \mathrm{L}$ syringe containing $10 \mu \mathrm{g} / \mu \mathrm{L}$ of ibotenic acid was then positioned at the coordinates for the basolateral amygdala at $3.8 \mathrm{~mm}$ lateral to midline, $1.0 \mathrm{~mm}$ posterior to bregma, and $6.0 \mathrm{~mm}$ underneath the skull at a $4^{\circ}$ angle. The ibotenic acid was dispensed in the amount of $10 \mu \mathrm{g} / \mu \mathrm{L}$ in a solution of $0.1 \mathrm{pH} 7.4$ phosphate-buffered saline from a microliter syringe. When the syringe tip was in place, the ibotenic acid was dispensed at $.15 \mu \mathrm{L} / \mathrm{min}$. After the ibotenic acid injection, the animals were placed in a recovery chamber until four minutes post-injection. After four minutes, the subjects were placed in their mother's container and were allowed to remain there until PND 25. At PND 25, the animals were weaned and then placed in stainless steel cages with same sex and same litter groups of three.

\section{Social Testing}

Social testing was based on and modified from Gittis \& Alfieri (2009). An ABCDEABCDE within subject design for treatment was implemented to observe the effects of OT, AVP, DCS, and saline on social behavior. For the first day of testing on PND 25, the subjects were randomly assigned to either the OT, AVP, DCS or saline treatment. OT was administered 120 minutes before social testing via a subcutaneous injection at a dose of .6 ng/kg. AVP was given 120 minutes before social testing via a subcutaneous injection at a dose of $10 \mathrm{mg} / \mathrm{kg}$. DCS was given 20 minutes prior to social testing in the amount of $320 \mathrm{mg} / \mathrm{kg}$. All lesioned subjects were matched in same sex pairs (male-male and female-female) with all the rats being paired with an animal of a different litter to prevent a pre-existing social familiarization. Social behavior in rats was defined as body contact, jumping over or running under one another, anogenital sniffing, social sniffing, cuddling, and social contact (Daenen, Wolterink, Gerrits, \& Van Ree, 2002). The social test involved two rats of the same sex being placed in a container for 300 seconds and the amount of social behavior exhibited by one of the rats was recorded. During social testing, all trials were recorded so the social behavior could be quantified in seconds by a team of coders that were trained by the researcher. The animals were tested on PND 25, PND 27, PND 29, PND 31, and PND 33.

The researcher was trained by Dr. Alan Gittis and Dr. Robin McGovern for every procedure employed in this study. In order to make sure all procedures were done ethically and correctly, Dr. Gittis and Dr. McGovern demonstrated and explained how to perform all procedures and observed initial protocol and procedures. The researcher also had research assistants available in order to aid with lesioning surgery and social testing. The two research assistants were trained by the researcher after the researcher became familiar with all procedures. Twelve individuals were trained by the researcher to code social behavior data. The twelve coders were split into four groups of three and each group was assigned social behavior videos to code. Coding social data consisted of quantifying in seconds the amount of social behavior exhibited by a given rat by reviewing a video of the matched animal pairs. Stop-watches were employed to facilitate recording the amount of time the subjects interacted. Those coding the social behavior were informed of the condition of a given rat in a matched pair so as to avoid bias. Additionally, the inter-rater reliability of the coders was also reviewed.

\section{Statistical Analysis}

A parallel correlation was chosen to assess the consistency of the inter-rater reliability of the coders in order to ensure that the data for the amount of social behavior was accurate. Furthermore, using a parallel correlation would also verify that the results obtained were not due to an error in coding the social behavior. A mixed between-within subjects analysis of variance test was used to compare the main effects of treatment on social behavior and the role of sex affecting treatment efficacy.

\section{Results}

A parallel correlation was conducted to measure the inter-rater reliability of the three coders for determining the amount of social behavior in seconds for each rat. The values for social interaction time among the coders were significantly positively correlated. There was a positive correlation and significance for the first coder compared to the second coder was $(r(107)=.932, p<.01)$ and the significance for the first coder compared to the third coder was $(r(107)=.860, p<.01)$. When the second coder was compared to the first coder a significance of $(r(107)=$ $.847, p<.01$ ) was found and was also positively correlated, meaning that the results obtained were not due to chance due to errors in coding. The mean of the coder ratings for social interaction times were used for analysis.

Thirty-two animals underwent the bilateral amygdala lesion surgery; however, only five males and sixteen females survived the lesioning surgery. One female rat was excluded from analysis due to a limited supply of AVP and was thus unable to receive the treatment. The predictions being examined were tested using a mixed between-within subjects analysis of variance.

The prediction that treatment affected the amount of social interaction was significant (Wilks' Lambda $=$ $\left..125, F(4,72)=2.49, p=.051, \eta^{2}=.122\right)$. Additionally, the main effect that the sex of the animals would affect treatment efficacy approached significance (Wilks' Lambda $\left.=.221, F(4,72)=2.33, p=.064, \eta^{2}=.115\right)$. This approaching significance is most likely due to a low number of male rats available. The lack of male subjects occurred because there were only seven male animals born out of the four litters. Furthermore, only five of the males survived surgery. Moreover, the means for both sexes varied dramatically by condition. These large variations in social behavior influenced the statistical analyses for treatment. Although the interaction between how sex affects treatment approaches significance, the sexually dimorphic differences in treatment can be observed in Figure 1. 
For the social interaction baseline, there was little variation in the amount of social behavior when the animals were administered the control condition saline. The prediction that OT would increase social behavior in females was non-significant, but females did show an increase in social behavior as compared to males, which showed a decrease in social behavior when given OT. In addition, upon administration of AVP to subjects, there was a non-significant increase in social behavior for male subjects. Males demonstrated an increase in social behavior when given AVP versus females, in which the subject's social behavior remained close to that of the baseline. Upon administration of DCS, social behavior increased for both sexes $\left(F(1,18)=8.85, p<.05, \eta^{2}=\right.$ .330). Furthermore, there was also a significant interaction that sex affected the amount of social time when given DCS $F(1,18)=5.41, p<.05, \eta^{2}=.231$. Although both sexes had a significant increase in social behavior for the DCS condition, males on average had a much larger increase in social interaction than did females. Lastly, when females were given DCS + OT, an attenuating effect on social behavior was observed. Males showed a small non-significant increase in social interaction. These results do not support the prediction that DCS + OT would increase social behavior significantly more than the other treatments.

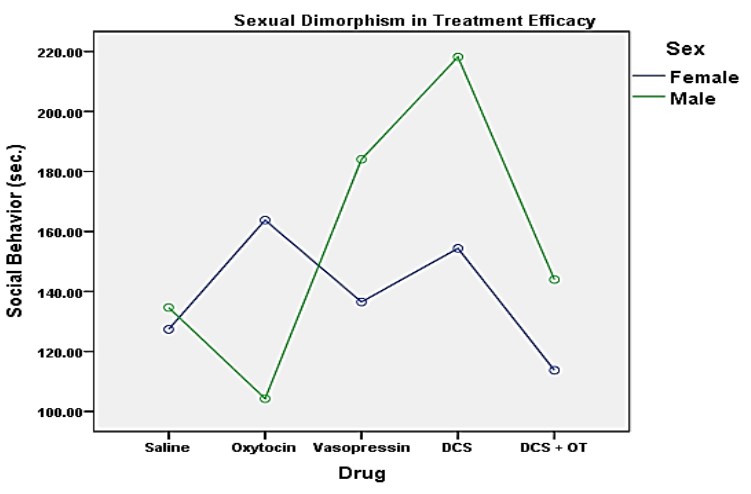

Figure 1: Differences treatment response due to sexual dimorphism. Social behavior is quantified in seconds for 21 male and female rats for 5 treatments.

\section{Discussion}

This study demonstrated that social behavior in rats with amygdalar lesions was altered upon administration of OT, AVP, DCS, and DCS + OT. Both increases and attenuations in the amount of social behavior were observed based on the treatment condition and sex of the animals. In addition, the differences in the amount of social behavior for a given treatment varied drastically upon comparison of both sexes, although the results were found to be approaching significance. Because only five male rats underwent testing due to surgery mortality as well as breeding difficulties, this low number of male subjects resulted in a lower power to test the interaction between drug condition and sex.

Even though the effect of OT was nonsignificant, there was an increase in social behavior for females. Furthermore, OT was found to be the most effective treatment for female rats with social deficits, whereas males experienced an attenuation in social behavior. The findings that OT increased social behavior in a rat bilateral amygdala lesion model were consistent with Gittis \& Alfieri's study in 2009. Additionally, the reduction of social behavior in male rats when administered OT may be explained the potential of OT to act as an antagonist to vasopressin (Carter, 2007). Because vasopressin is the male analogue responsible for social and affiliative behavior in males, it could be reasonably concluded that the antagonistic effect of OT could have negatively affected social behavior in males. Similar results that OT can reduce social behavior in males were also found in Dantzer, Bluthe, Koob, \& Le Moal's study (1987), who suggested that OT could cause social recognition deficits in male rats.

The influence of AVP on social behavior was similarly non-significant, but variance in the amount of social behavior between the sexes was also observed. AVP was the second most effective-treatment for reversing the social deficit present in the male animals. The observation that AVP increased social behavior was supported by Dluzen, Muraoka, Engelmann, \& Landgraf (1998) and by Dantzer, Bluthe, Koob, \& Le Moal's study (1987). In addition, because males possess more vasopressin receptors than females do, one could conclude that males are more responsive to AVP than are females (Carter, 2007). Female subjects, on the other hand, only experienced a minor increase in social behavior from the control condition. According to Carter (2007), male hormones such as testosterone may have the capability to change how the body reacts to OT or alter OT's function. Since vasopressin's precursor is testosterone, the remnant of OT being produced outside the confines of the amygdala within the brain may have been offset by the introduction of exogenous AVP.

Despite OT and AVP's non-significant influence on behavior, DCS significantly reversed the social deficits in both sexes. However, males demonstrated an increase in their social behavior from baseline by 28 percent versus females, who experienced a 9 percent increase in their amount of social behavior. DCS was found to be the most beneficial therapeutic agent for males in treating social impairment within this study. Moreover, a large difference in the amount social behavior was observed when comparing males and females. This difference in response to DCS was due to the sexually dimorphic properties of the NMDA receptors in the brain (Kirkpatrick \& Kakoyannis, 2004; Cyr et al., 2001). NMDA receptors have been implicated in ASDs by virtue of the lack of sociability exhibited in transgenic mice that have a mutation in the NR1 subunit of this receptor (Jacome, Burket, Herndon, \& Deutsch, 2011). The mechanism by which DCS may act enhances the rate that social information is acquired by operating as an agonist to the NMDA receptors and supposedly aids in OT binding (Modi \& Young, 2011; Jacome, Burket, Herndon, \& Deutsch, 2011). However, little is known about the actual mechanism by which DCS works. DCS may also facilitate the binding of vasopressin as well since both OT and AVP are very closely related. Hence, males may have experienced a larger increase in 
social behavior because DCS may facilitate the interactions between vasopressin and its receptors.

In contrast, when the subjects were given DCS + OT, a slight increase in the amount of social behavior was present in the population of males as compared to the female rats, who experienced an attenuation in social behavior. The increase in male social behavior was due to DCS's property of improving the amount of social behavior was just enough to counteract OT's attenuating effect on social behavior. As for females, even though DCS by itself increased social behavior, the addition of OT paradoxically decreased social behavior. One reason why social behavior might have been impaired in females could have been due to DCS's property of aiding in OT binding since OT can have an attenuating effect when present in more than optimal doses (Gittis \& Alfieri, 2009; Modi \& Young, 2011; Otto, 2011). In essence, DCS could have promoted the binding of exogenous OT to the point at which OT began to have a detrimental effect on the amount of social behavior.

This study demonstrated that DCS reversed the social deficits, which are analogous to the social impairment found in ASDs and should be further investigated as a therapeutic agent. Additionally, the mechanism by which DCS operates should be further explored to aid in understanding how to properly treat social deficits such as determining if DCS interacts with any other compounds besides possibly OT. Furthermore, the sex of the subject affects treatment efficacy on the amount of social behavior observed. Thus, sexually dimorphic treatments for social impairments should continue to be researched.

\section{Bibliography}

Al-Ayadhi, L. (2005). Altered oxytocin and vasopressin levels in autistic children in Central Saudi Arabia. Neurosciences 10, 47-50.

Amaral, D., Bauman, M., \& Schumann, C. (2003). The amygdala and autism: implications from non-human primate studies. Genes Brain and

Behavior, 2(5), 295-302. doi: 10.1046/j.1601183X.2003.00043.x

American Psychiatric Association. (2001). Diagnostic and statistical manual of mental disorders

( $4^{\text {th }}$ ed., text rev.). Retreived from http://www.dsm5.org/ProposedRevisions/Pages/p roposedrevision .aspx ?rid=94

Bachevalier, J., \& Loveland, K. (2006). The orbitofrontalamygdala circuit and self-regulation of socialemotional behavior in autism. Neuroscience and Biobehavioral Reviews, 30(1), 97-117. doi: 10.1016/j.neubiorev.2005.07.002

Bachevalier, J., \& Malkova, L. (2006). The amygdala and development of social cognition:

Theoretical comment on Bauman, Toscano, Mason, Lavenex, and Amaral (2006). Behavioral Neuroscience, 120(4), 989-991. doi: 10.1037/0735-7044.120.4.989

Baron-Cohen, S. (2002). The extreme male brain theory of autism. Trends in Cognitive Sciences, 6(6), 248-254.
Bartz, J., \& Hollander, E. (2008). Oxytocin and experimental therapeutics in autism spectrum disorders. In I. D. Neumann \& R. Landgraf (Eds.), Advances in Vasopressin and Oxytocin: from Genes to Behaviour to Disease, 170, 451462). Amsterdam: Elsevier Science Bv.

Bielsky, I., Hu, S., Szegda, K., Westphal, H., \& Young, L. (2004). Profound impairment in social recognition and reduction in anxiety-like behavior in vasopressin V1a receptor knockout mice. Neuropsychopharmacology, 29(3), 483493. doi: 10.1038/sj.npp.1300360

Bielsky, I., Hu, S., \& Young, L. (2005). Sexual dimorphism in the vasopressin system: Lack of an altered behavioral phenotype in female V1a receptor knockout mice. Behavioural Brain Research, 164(1), 132-136. doi: 10.1016/j.bbr.2005.06.005

Bielsky, I., \& Young, L. (2004). Oxytocin, vasopressin, and social recognition in mammals. Peptides, 25(9), 1565-1574. doi: 10.1016/j.peptides.2004.05.019

Bill, B., \& Geschwind, D. (2009). Genetic advances in autism: heterogeneity and convergence on shared pathways. Current Opinion in Genetics \& Development, 19(3), 271-278. doi: 10.1016/j.gde.2009.04.004

Cardinal, R., Parkinson, J., Hall, J., \& Everitt, B. (2002). Emotion and motivation: the role of the amygdala, ventral striatum, and prefrontal cortex. Neuroscience and Biobehavioral Reviews, 26(3), 321-352.

Carter, C. (2007). Sex differences in oxytocin and vasopressin: Implications for autism spectrum disorders? Behavioural Brain Research, 176(1), 170-186. doi: 10.1016/j.bbr.2006.08.025

Cyr, M., Ghribi, O., Thibault, C., Morissette, M., Landry, M., \& Di Paolo, T. (2001). Ovarian steroids and selective estrogen receptor modulators activity on rat brain NMDA and AMPA receptors. Brain Research Reviews, 37(13), 153-161. doi: 10.1016/s0165-0173(01)001151

Daenen, E., Wolterink, G., Gerrits, M., \& Van Ree, J. (2002). The effects of neonatal lesions in the amygdala or ventral hippocampus on social behaviour later in life. Behavioural Brain Research, 136(2), 571-582.

Dantzer, R., Bluthe, R., Koob, G., \& Le Moal, M. (1987). Modulation of social memory in male rats by neurohypophyseal peptides. Psychopharmacology (Berl), 91(3), 363-368.

Davis, M., \& Whalen, P. (2001). The amygdala: vigilance and emotion. Molecular Psychiatry, 6(1), 13-34.

Dluzen, D., Muraoka, S., Engelmann, M., \& Landgraf, R. (1998). The effects of infusion of arginine vasopressin, oxytocin, or their antagonists into the olfactory bulb upon social recognition responses in male rats. Peptides, 19(6), 999-1005. doi: 10.1016/s01969781(98)00047-3 
Frank, E., \& Landgraf, R. (2008). The vasopressin system From antidiuresis to psychopathology. European Journal of Pharmacology, 583(2-3), 226-242. doi: 10.1016/j.ejphar.2007.11.063

Gittis, A., \& Alfieri, A. (2009). The role of oxytocin in facilitating social behavior in rats with amygdala lesions [Abstract]. Society for Neuroscience Abstracts, 463.16.

Green, L., Fein, D., Modahl, C., Feinstein, C., Waterhouse, L., \& Morris, M. (2001). Oxytocin

and autistic disorder: Alterations in peptide forms. Biological Psychiatry, 50(8), 609-613.

Hamann, S., \& Adolphs, R. (1999). Normal recognition of emotional similarity between facial expressions following bilateral amygdala damage. Neuropsychologia, 37(10), 1135-1141.

Hammock, E., \& Young, L. (2006). Oxytocin, vasopressin and pair bonding: implications for autism. Philosophical Transactions of the Royal Society B-Biological Sciences, 361(1476), 21872198. doi: $10.1098 /$ rstb.2006.1939

Jacome, L., Burket, J, Herndon, A., \& Deutsch, S. (2011). D-cycloserine enhances social exploration in the balb/c mouse. Brain Research Bulletin, 85(3-4), 141-144. doi: 10.1016/j.brainresbull.2011.03.004

Kemper, T., \& Bauman, M. (1993). The contribution of neuropathologic studies to the understanding of autism. Neurologic Clinics, 11(1), 175-187.

Kirkpatrick, B., \& Kakoyannis, A. (2004). Sexual dimorphism and the NMDA receptor in alloparental behavior in juvenile prairie voles (Microtusochrogaster). Behavioral Neuroscience,
$118(3), \quad 584-589$ doi: $10.1037 / 0735-$ 7044.118.3.584

Lim, M., Bielsky, I., \& Young, L. (2005). Neuropeptides and the social brain: potential rodent models of autism. International Journal of Developmental Neuroscience, 23(2-3), 235-243. doi: 10.1016/j.ijdevneu.2004.05.006

McDougle, C., Stigler, K., Erickson, C., \& Posey, D. (2006). Pharmacology of autism. Clinical Neuroscience Research, 6(3-4), 179-188. doi: 10.1016/j.cnr.2006.06.012

Modi, M., \& Young, L. (2011). D-cycloserine facilitates socially reinforced learning in an animal model relevant to autism spectrum disorders. Biological Psychiatry, 70(3), 298-304. doi: 10.1016/j.biopsych.2011.01.026

Otto, M. (2011). Expanding findings on d-cycloserine augmentation of therapeutic learning: A role for social learning relative to autism spectrum disorders? Biological Psychiatry, 70(3), 210-211. doi: 10.1016/j.biopsych.2011.06.005

Posey, D., Kem, D., Swiezy, N., Sweeten, T., Wiegand, R., $\&$ McDougle, C. (2004). A pilot study of d-cyloserine in subjects with autistic disorder. American Journal of Psychiatry, 161(11), 2115-2117. doi: 10.1176/appi.ajp.161.11.2115

Schanen, N. (2006). Epigenetics of autism spectrum disorders. Human Molecular Genetics, 15, R138-R150. doi: 10.1093/hmg/dd1213 